\title{
Downscaling of Short-Term Precipitation Time Series for Climate Change Impact Assessment
}

\author{
Jonas Olsson $^{1}$, Lars Gidhagen ${ }^{1}$, and Akira Kawamura ${ }^{2}$ \\ ${ }^{1}$ Swedish Meteorological and Hydrological Institute, 60176 Norrköping, Sweden \\ 2 Tokyo Metropolitan University, 1-1 Minami-Osawa, Hachioji, Tokyo 192-0397, Japan \\ jonas.olsson@smhi.se, lars.gidhagen@smhi.se, \\ akira.kawamura@tmu.ac.jp
}

\begin{abstract}
A future increase of short-term precipitation intensities may lead to problems in sewer systems, such as increased overflow volumes and flood risks. To quantify the consequences, downscaling of climate model precipitation is required to the scales relevant in urban hydrology. In the SUDPLAN project, a system where users may upload historical time series to be used as a basis for such downscaling is being developed. In this paper, the method (Delta Change) is outlined along with brief descriptions of the technical solution and result visualization.
\end{abstract}

Keywords: climate model, urban hydrology, SUDPLAN.

\section{Introduction}

Precipitation is often accumulated in time to days, months or years, but in some cases the short-term rainfall is the most interesting. With short-term we mean rainfall observed with a very short time step, e.g. 1 minute or even finer. In contrast to long-term accumulations, which represent averages over long periods, short-term precipitation thus corresponds to the instantaneous intensity (and intensity changes) that we perceive during a storm event.

Short-term precipitation is of particular interest in urban hydrology. Because of the large fraction of impervious surface in the urban environment the runoff process is very fast. Storm water moves rapidly on the surface to a sewer system inlet after which the flow is concentrated in the sewer pipe network. To simulate the flow response to an actual storm in a computer model of the urban environment, a time step of $1 \mathrm{~min}$ or shorter is required to capture the variability of the runoff. Thus also the input, i.e. precipitation time series, needs to have a very high time resolution. Such observations are commonly obtained using a tipping bucket gauge that registers a time stamp every time a small amount of rainfall (e.g. $0.2 \mathrm{~mm}$ ) has accumulated. Further, urban catchments are generally very small, on the order of $1 \mathrm{~km}^{2}$, so typically observations from only one gauge is used as input.

Short-term precipitation intensities are expected to increase as a function of the global warming. Both theoretical considerations [1] and climate model projections [2, 3] generally support this hypothesis. This implies an increased load on sewer systems with potential consequences in terms of increased overflow volumes and flood risks 
[4]. To estimate the consequences quantitatively, sewer model simulations with precipitation input representing the future climate are required. A key problem, however, is that the spatial (and, to a lesser degree, also the temporal) resolution of Regional Climate Models (RCMs) is too coarse for directly applying the precipitation results in sewer system models. Climate model results represent a mean precipitation over an area of typically 1000-2000 $\mathrm{km}^{2}$ whereas sewer models, as mentioned above, needs precipitation observed in a single location. Thus, some form of downscaling from the climate model grid scale to the local grid scale is required [3, 4].

In this paper, the method and system for short-term precipitation downscaling used within the project SUDPLAN (Sustainable Urban Development Planner for Climate Change Adaptation) is described. The downscaling is based on the Delta Change concept, i.e. a rescaling of historical observations in line with the expected future changes. The technical solution as well as result visualization is briefly described.

\section{Downscaling Method: Delta Change}

Precipitation time series downscaling is performed by Delta Change, which generally means that future changes of some climate variable (e.g. precipitation or temperature), as estimated from climate model data, are transferred to historical observations of the same variable [5]. In the SUDPLAN application, future changes of the frequency distribution of 30-min precipitation intensities, which is the shortest time step available in climate model data, are transferred to historical short-term rainfall observations.

After the historical time series to be rescaled has been defined, the first step in the procedure is to extract 30-min precipitation time series from climate model projections. Data from two 30-year periods are used; one reference period centered over the historical time series and one selected future period. The frequency distribution of the precipitation intensities in each period is calculated, after which the ratio of each future percentiles to the same reference percentile is calculated. This ratio is called Delta Change Factor (DCF; $>1$ implies increase, and vice versa).

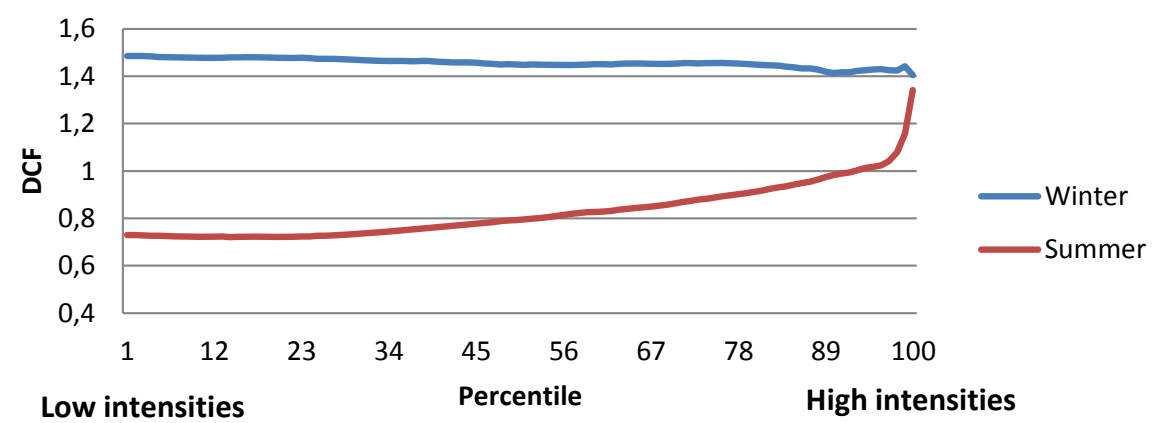

Fig. 1. Example of future changes of rainfall intensities as estimated from a Regional Climate Model. $\mathrm{DCF}=1$ implies no change, $\mathrm{DCF}<1$ a future decrease and $\mathrm{DCF}>1$ a future increase. 
Fig. 1 shows an example of DCFs and illustrates how this approach makes it possible to handle rather complicated future changes, such as increased high intensities but decreased intermediate and low intensities (summer in Fig. 1). Fig. 1 also demonstrates the need for performing the downscaling on a seasonal basis, as future changes may be radically different for different seasons. It should be mentioned that the methodology requires an assumption that DCFs estimated from data on the RCM resolutions in time and space are valid also for the higher resolutions of the historical observations.

Finally, the calculated DCFs may be transferred to the historical observations. This is achieved by firstly aggregating the observations to a 30-min time step, to correspond with the climate model output. Secondly a frequency analysis is performed to identify each 30-min value's corresponding percentile and, in turn, DCF. In the last step, the volume of each registration is rescaled in line with the DCF of the corresponding 30-min value. For further details on the approach, see [3].

\section{Technical Solution}

In SUDPLAN, the data flows and model executions involved in the downscaling are managed by a Scenario Management System (SMS) and a core of Common Services (CS), where the latter includes modules for downscaling of not only precipitation but also air quality and hydrology (Fig. 2). Two types of user specifications are needed to launch a model simulation. (1) CS receives from the SMS the coordinates for the station, the data from which are to be downscaled, together with some identification

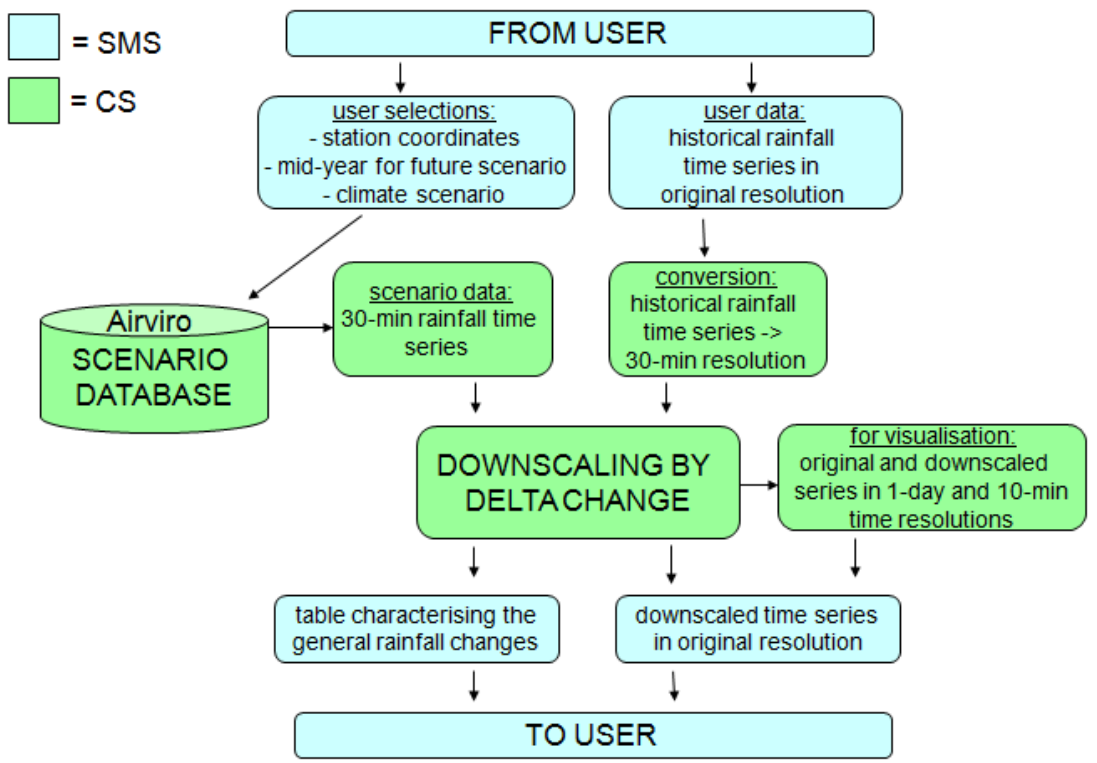

Fig. 2. Components of and data flows between Scenario Management System (SMS) and Common Services (CS) for precipitation downscaling 
of the coordinate system and map projection. The user has also specified which climate scenario to use and which future 30-year time period to perform the downscaling for. (2) Short-term rainfall observations from a tipping-bucket gauge or equivalent on a specified format, time stamp (resolution: minute or higher) followed by a rainfall registration $(\mathrm{mm})$.

The model execution includes the following steps. (1) Extraction of 30-min rainfall time series from the CS Scenario Database, in five RCM grid boxes surrounding the station. (2) Conversion of historical time series to 30-min time resolution, to be compatible with scenario data. (3) Downscaling by Delta Change, including ( $i$ ) estimation of future changes from the scenario data and (ii) transfer of the estimated changes to the historical time series. (4) Calculation of general rainfall changes from reference period to scenario period. (5) Conversion of both historical and downscaled time series to 1-day and 10-min time resolutions, for visualization.

\section{Output and Visualization}

The main output is a time series which differs from the uploaded one in two ways. (1) In each time stamp, the observation year has been replaced by a future target year. (2) Each rainfall registration has been re-scaled to account for future changes in the probability distribution of short-term rainfall intensities (Fig. 3).

The automated CS downscaling function has been developed and verified using short-term rainfall data from Kalmar, Sweden, that are well known to the developer and thus optimal for this purpose. Figure 3 shows the first part of the original and downscaled time series. In this data, the gauge resolution was $0.2 \mathrm{~mm}$; thus all original registrations have this value. In the downscaling procedure, the gauge resolution is assumed variable and modified to account for the climate change impact. In this application, a target period 70 years ahead was used, which is reflected in the time stamps (Fig. 3).

\begin{tabular}{|l|l|l|l|}
\hline \multicolumn{2}{|c|}{ ORIGINAL TIME SERIES } & \multicolumn{3}{|c|}{ DOWNSCALED TIME SERIES } \\
\hline $1991-10-0119: 04$ & 0,2 & $2061-10-0119: 04$ & 0,20666 \\
\hline $1991-10-0119: 11$ & 0,2 & $2061-10-0119: 11$ & 0,20666 \\
\hline $1991-10-0119: 15$ & 0,2 & $2061-10-0119: 15$ & 0,20666 \\
\hline $1991-10-0119: 30$ & 0,2 & $2061-10-0119: 30$ & 0,19427 \\
\hline $1991-10-0119: 39$ & 0,2 & $2061-10-0119: 39$ & 0,19427 \\
\hline $1991-10-0121: 04$ & 0,2 & $2061-10-0121: 04$ & 0,19427 \\
\hline $1991-10-0214: 17$ & 0,2 & $2061-10-0214: 17$ & 0,20594 \\
\hline $1991-10-0214: 18$ & 0,2 & $2061-10-0214: 18$ & 0,20594 \\
\hline $1991-10-0214: 18$ & 0,2 & $2061-10-0214: 18$ & 0,20594 \\
\hline $1991-10-0214: 19$ & 0,2 & $2061-10-0214: 19$ & 0,20594 \\
\hline
\end{tabular}

Fig. 3. Examples of original and downscaled time series

Besides the time series, the user will be provided with a table of percentage changes in different key rainfall properties (mean, maximum and frequency), separated into seasons. Further, visualization as outlined in Fig. 4 is suggested. The upper 


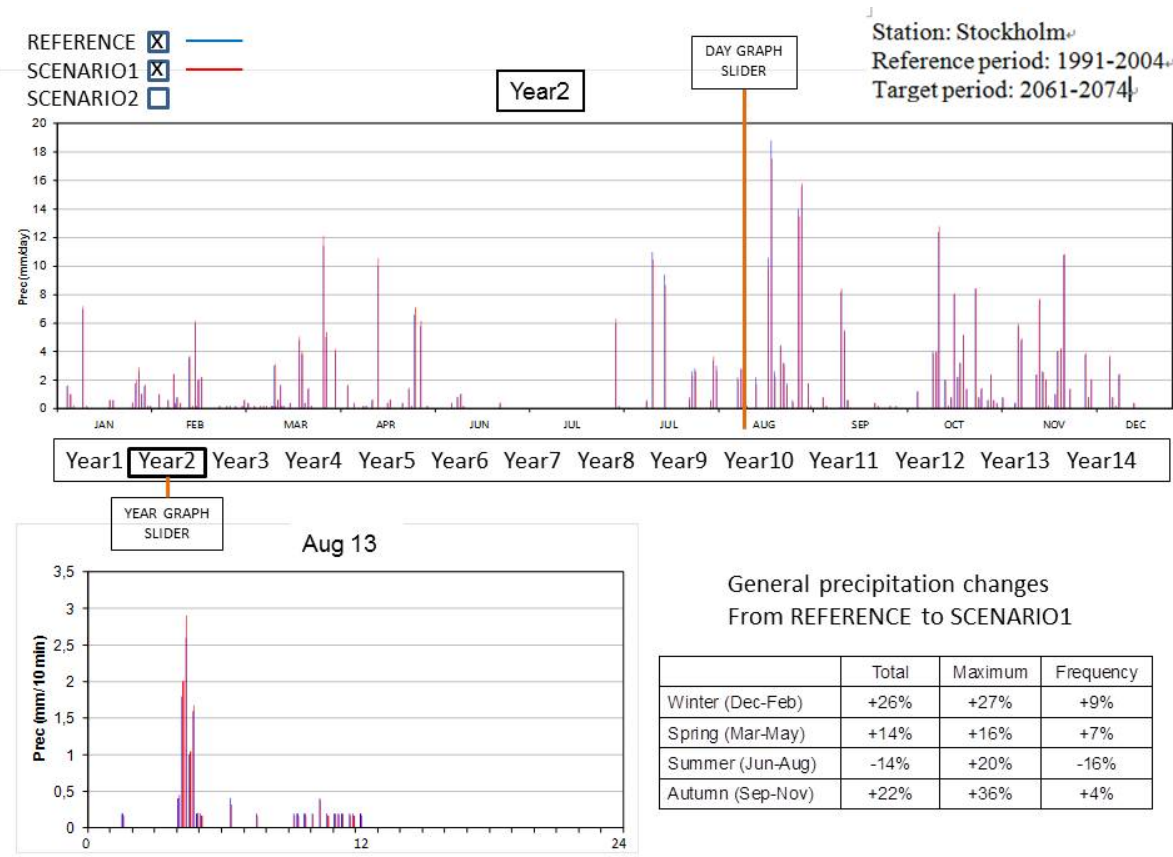

Fig. 4. Suggested approach for short-term rainfall time series visualization

graph shows daily rainfall on an annual basis, where the year can be selected using a 'year graph slider' below the graph. In the annual graph, by a 'day graph slider' a particular day can be selected and in the lower left diagram, 10-min rainfall during the selected date is shown. If both a reference and a scenario time series are selected, the lower right table shows the general rainfall changes from the reference to the scenario.

\section{Concluding Remarks and Future Prospects}

The precipitation time series downscaling functionality in SUDPLAN makes it possible for e.g. sanitation engineers and urban planners to obtain data for climate local change impact assessment studies. The methodology based on Delta Change (DC) a straight-forward and not without limitations. One general limitation is that the downscaled data will be strongly influenced by the properties of the historical data, with respect to e.g. variability. Another limitation is that any change in precipitation frequency is not taken into account in DC-based downscaling. Despite these limitations, in our opinion a DC-based approach is the only practicable solution in an automated system like this.

Future work will include development of the DC-approach to also be able to handle frequency changes. Preliminary analyses indicate that future precipitation frequency changes are generally caused by a change in the number of precipitation events rather than changes in event durations. Thus it may be possible to either 
remove or multiply observed events, in a clever way, to simulate frequency changes. On a more general level, the spatial resolution of RCMs is rapidly increasing and already results at $\sim 10 \times 10 \mathrm{~km}$ and even higher are available. Thus the scale of simulated precipitation is approaching the point scale, conceivably reducing (and ultimately, hopefully, eliminating) the need for subsequent downscaling. Evaluating high-resolution RCM precipitation is thus an important area of future research.

Acknowledgments. The study was mainly performed within the SUDPLAN project (EU FP7 ICT-2009-6.4). Additional funding was provided by Tokyo Metropolitan University and the Swedish Research Council Formas, the latter through project HYDROIMPACTS2.0.

\section{References}

1. Trenberth, K.E., Dai, A., Rasmussen, R.M., Parsons, D.B.: The changing character of precipitation. Bull. Am. Meteorol. Soc. 84, 1205-1217 (2003)

2. Larsen, A.N., Gregersen, I.B., Christensen, O.B., Linde, J.J., Mikkelsen, P.S.: Potential future increase in extreme one-hour precipitation events over Europe due to climate change. Water Sci. Technol. 60, 2205-2216 (2009)

3. Olsson, J., Berggren, K., Olofsson, M., Viklander, M.: Applying climate model precipitation scenarios for urban hydrological assessment: A case study in Kalmar City, Sweden. Atm. Res. 92, 364-375 (2009)

4. Semadeni-Davies, A., Hernebring, C., Svensson, G., Gustafsson, L.-G.: The impacts of climate change and urbanisation on drainage in Helsingborg, Sweden: Suburban stormwater. J. Hydrol. 350, 114-125 (2008)

5. Hay, L.E., Wilby, R.L., Leavesley, G.H.: A comparison of delta change and downscaled GCM scenarios for three mountainous basins in the United States. J. Am. Water Resour. Assoc. 36, 387-397 (2000) 